JOURNAL OF SYNCHROTRON RADIATION

ISSN 1600-5775

Received 6 June 2016

Accepted 4 August 2016

Edited by M. Yabashi, RIKEN SPring-8 Center, Japan

Keywords: FEL; pump-probe experiment; user laser.

\section{The SwissFEL Experimental Laser facility}

\author{
Christian Erny* and Christoph Peter Hauri
}

SwissFEL, Paul Scherrer Institute, Villigen PSI 5232, Switzerland. *Correspondence e-mail: christian.erny@psi.ch

The hard X-ray laser SwissFEL at the Paul Scherrer Institute is currently being commissioned and will soon become available for users. In the current article the laser facility is presented, an integral part of the user facility, as most timeresolved experiments will require a versatile optical laser infrastructure and precise information about the relative delay between the X-ray and optical pulse. The important key parameters are a high availability and long-term stability while providing advanced laser performance in the wavelength range from ultraviolet to terahertz. The concept of integrating a Ti:sapphire laser amplifier system with subsequent frequency conversion stages and drift compensation into the SwissFEL facility environment for successful $24 \mathrm{~h} / 7 \mathrm{~d}$ user operation is described.

\section{Introduction}

X-ray free-electron lasers (XFELs) have become the favourite research tool for many modern research fields, such as ultrafast magnetism (Kubacka et al., 2014; Rettig et al., 2015), nonlinear X-ray science (Glover et al., 2012; Shwartz et al., 2014), femto-chemistry (Kim et al., 2015; Zewail, 2000), biology (Barty et al., 2012), warm-matter physics (Lévy et al., 2015), atomic physics (Young et al., 2010) and many more. Currently SwissFEL is being commissioned at the Paul Scherrer Institute in Switzerland and will become online by the end of 2017 for user operation. The machine will provide hard X-ray radiation of 2-12 keV with pulse durations tunable between $50 \mathrm{fs}$ and $5 \mathrm{fs}$ (Ganter, 2010). This operation mode can be extended to the attosecond regime, to the broadband emission mode or to the high-peak-power (TW) regime (Prat et al., 2015; Prat \& Reiche, 2015).

One of the main advantages of SwissFEL is the excellent timing system which will provide a synchronization of all timecritical components with an expected timing jitter of less than 10 fs r.m.s (Ganter, 2010). This renders SwissFEL highly attractive for time-resolved experiments with femtosecond resolution. For successful time-resolved experiments the performance of the optical laser, acting as pump or probe, is as equally important as the free-electron laser (FEL) source. Best temporal resolution is achieved when the probe duration is similar to or shorter than the pump pulse duration and when the arrival time between the two pulses is precisely known. The optical laser system should thus not only provide short pulses for the experiment but also serve as a reference for $\mathrm{X}$-ray arrival-time diagnostics.

In the first phase, SwissFEL will operate with two endstations (ESA and ESB). Both endstations are optimized for $\mathrm{X}$-ray pump-probe experiments. In detail, ESA is equipped with a liquid-jet sample manipulator and a van Hamos-type spectrometer and optimized for pump-probe X-ray absorption spectroscopy, X-ray emissions spectroscopy, including 
resonant inelastic X-ray scattering, X-ray diffuse scattering and femtosecond serial nanocrystallography (Milne, 2013).

ESB is optimized to study the time-dependent behaviour of correlated materials in condensed matter. For this it includes a general-purpose heavy-load sample stage with a robot detector arm, as well as a heavy-load diffractometer with detector arm, allowing for time-resolved X-ray diffraction, time-resolved inelastic $\mathrm{X}$-ray scattering and many more techniques (Ingold \& Beaud, 2013).

Depending on the experimental sample, the optical laser needs to be tuned over a large spectral bandwidth ranging from the ultraviolet (UV) (Budarz et al., 2016), to the visible (Lemke et al., 2013), the infrared (IR) (Bertoni et al., 2015), the mid-IR (Mankowsky et al., 2014), up to the THz range (Kubacka et al., 2014). As FEL beam time is precious and needs to be used efficiently, the optical laser availability must not be affected by the high versatility. This requires, for dayto-day user operation, a high availability of the optical pump laser, ideally $24 \mathrm{~h} / 7 \mathrm{~d}$. Also the switching time between experiments must be used efficiently and kept short. Time and effort required to prepare the laser for a new experiment must be as short as possible. The users thus require a laser system with the best available performance, the highest flexibility and latest technology, while, from the operations side, a high availability with low maintenance requirements is desired. In general, all these requirements are difficult to fulfil simultaneously, without compromises or significant development and advanced engineering.

This paper presents the technical solution chosen at the SwissFEL Experimental Laser facility for matching all these requirements. At its heart is a Ti:sapphire amplifier system which includes several fixed installed frequency conversion modules. We discuss the integration of the Ti:sapphire technology for reliable user operation and cutting-edge experiments and give an overview of the optical performance.

\section{Core laser system}

The Ti:sapphire amplifier systems of the SwissFEL Experimental Laser facility are located directly above the endstations (Laser I and Laser II, Fig. 1). The dedicated laser room (LHx) is an ISO class 7 clean room with a temperature stability of $\pm 0.1^{\circ} \mathrm{C}$ and a relative humidity of $40-45 \%$. The laser tables are placed inside a laser enclosure to shield the

\section{LHx Experimental laser room}

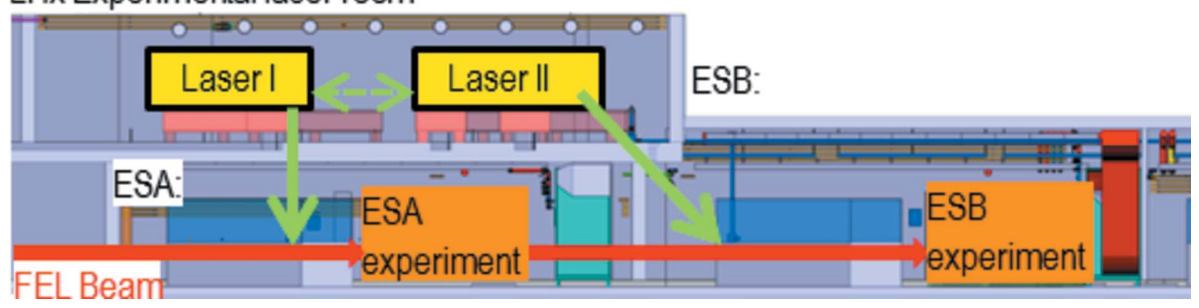

Figure 1

Layout of the experimental stations ESA and ESB with the pump laser hutch LHx on top. Laser I and Laser II assure redundancy and reliable operation. Evacuated transfer lines are installed for the beam transport between LHx and the endstations. laser from residual dust and turbulences in air. The laser power supplies are placed in water-cooled racks to reduce the heat load and air flow inside the laboratory. Laser I and Laser II have identical performance. During normal operation each laser system is dedicated to an individual endstation (Laser I for ESA and Laser II for ESB). This allows laser operation at both endstations simultaneously, for alignment and preliminary tests before the actual X-ray beam time starts. The redundant layout allows both lasers to be redirected to either endstation. This minimizes the risk of downtime of an experiment due to potential laser failure.

Pulse compressors, frequency conversion stages, delay lines and diagnostic tools are installed on the optical table close to the X-ray experimental instrument at the endstations. The beam transport between LHx and the endstations is realised by sending the uncompressed pulse through evacuated pipes (vacuum level $<10^{-4}$ mbar) and uses an optimized beam size for 3-inch optics. This keeps the pulse intensity low ( $<50 \mathrm{MW} \mathrm{cm}^{-2}$ outside and $<25 \mathrm{GW} \mathrm{cm}^{-2}$ at the focus) and prevents pulse distortions through nonlinear interaction and self-phase modulation. The output from the amplifier is relay imaged to the compressor with an intermediate focus inside the transfer line. Eventually a beam pointing stabilization between LHx and the endstations can be installed. Similar to LHx, the laser tables will be enclosed. The temperature stability inside the endstations is only controlled to $\pm 0.5^{\circ} \mathrm{C}$. Additional thermal isolation panels will improve the thermal stability on the optical tables, according to simulations to $\pm 0.1^{\circ} \mathrm{C}$. At the same time they act as a laser safety enclosure and allow laser operation inside the laser enclosure while the endstation is accessible.

The core laser system is based on mature chirped-pulse amplification Ti:sapphire technology which is commercially available from Coherent. The decision for this approach was based on a risk analysis. Ti:sapphire is the most used gain material for laser amplifiers providing pulse durations of less than 30 fs with significant pulse energy. Furthermore, various techniques have been developed to expand its spectral range towards the UV and the IR, to perform temporal and spatial pulse shaping and to control the actual electric field through CEP stabilization (Telle et al., 1999). In recent years, a large effort has been undertaken by assemblers to fulfil industrial standards for Ti:sapphire technology, resulting in unprecedented availability and reliability of today's system. The SwissFEL Experimental Laser is based on the Vitara Oscillator, Legend Elite Duo HE+ amplifier, with a custommade post-amplifier stage and Revolution pump lasers. With this layout it provides a compressed output energy of $>20 \mathrm{~mJ}$, centred at $800 \mathrm{~nm}$ and at $100 \mathrm{~Hz}$ repetition rate. The laser energy is divided into several beams to serve the experiment and the numerous X-ray pulse characterization, arrival time and drift compensation tools, including the $\mathrm{THz}$ streak camera (Juranić et al., 
2014b) and the laser arrival-time monitor (LAM) (Csatari Divall et al., 2015) (Fig. 2a).

The nominal laser pulse duration is $30 \mathrm{fs}$. This is in between the X-ray pulse duration of the high charge mode (50 fs) and the low charge mode $(<10 \mathrm{fs})$. In principle, shorter pulse duration at similar output energy has been demonstrated using Ti:sapphire amplifiers (Trisorio et al., 2011) or optical parametric chirped pulse amplification (Stanislauskas et al., 2014; Tavella et al., 2007); but this comes at the cost of increased complexity, higher maintenance efforts and increased risk of failure during operation. In either case, a large development effort is required to make these systems compatible with $24 \mathrm{~h} / 7 \mathrm{~d}$ user operation. Already the dispersion management of the beam transport between amplifier and experiment over a long distance is challenging.

The most critical parts in a laser amplifier system are the seed oscillator and pump lasers. The oscillator (Vitara-T) provides up to $125 \mathrm{~nm}$ bandwidth with $>400 \mathrm{~mW}$ output power. It is fully automated for long-term hands-free opera-

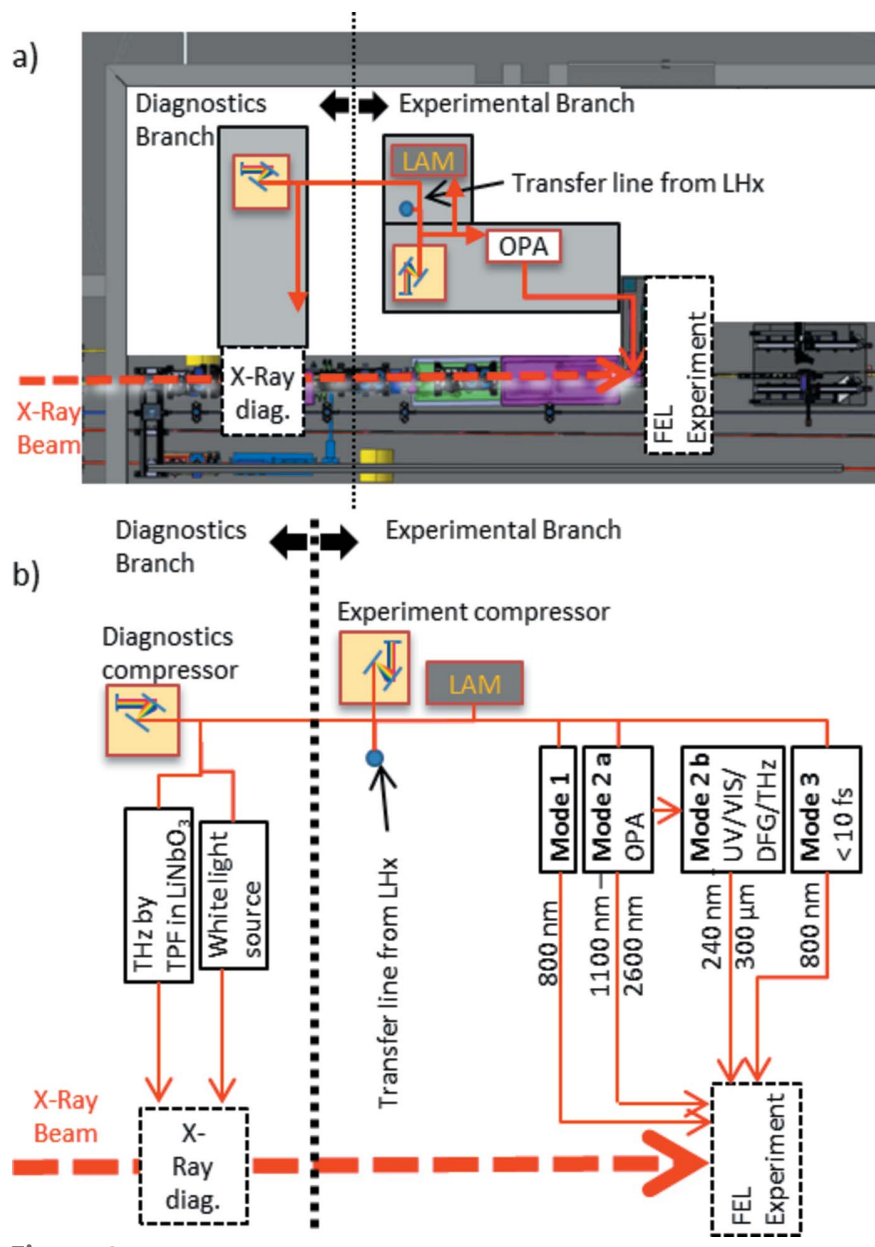

Figure 2

(a) General layout of the optical setup in the endstations. The beam is split into two branches (Diagnostics and Experimental). Each branch is on a separate laser table and equipped with an individual compressor. The LAM is installed on the experimental branch. (b) Detailed layout of the standard user-available wavelength options: Mode 1, direct $800 \mathrm{~nm}$ beam; Mode 2a, near-IR (NIR) optical parametric amplifier (OPA); Mode 2b, NIR OPA together with additional frequency conversion stage; Mode 3, sub-10 fs sourced, based on hollow core fibre technology. tion, including automatic mode locking and guaranteed output power. The pump lasers (Revolution, based on the former Evolution) provide up to $45 \mathrm{~mJ}$ at $527 \mathrm{~nm}$ and $100 \mathrm{~Hz}$, and a beam profile optimized for amplifier pumping. Continuouswave pumping with diodes assures low optical noise. These products, including their predecessors, have been on the market for many years. Thus the production processes are well controlled and the risk of failure during operation are minimized. Furthermore, they have been successfully integrated at most existing FEL facilities (Danailov et al., 2011; Minitti et al., 2015; Tono et al., 2013).

In summary, the Ti:sapphire technology has proven to be very versatile and, due to its widespread use in research, the solution for many user demands are commercially available or have already been demonstrated. This reduces development time and the effort to implement new operation modes. All the components have a proven track record and are from the same provider. This simplifies maintenance and avoids risk which would occur by interfacing products from several companies. The optical layout is reduced to the minimum. This facilitates daily operation and maintenance, which reduces the risk of downtime due to time-consuming alignment procedures. For SwissFEL the presented solution provides the best compromise between reliability, performance and cost. It offers the best compromise between pulse duration and reliability with state-of-the-art Ti:sapphire technology.

\section{Expected performance at the endstations}

The conceptual layout of both endstations is a priori identical (Fig. $2 a$, shown for ESA; ESB has a slightly modified table arrangement). Upon arrival in the endstations the uncompressed laser beam is split into two branches, the so-called diagnostics branch and the experimental branch. Each branch is equipped with its individual compressor, such that chirp and compression can be controlled individually. All compressors are designed to handle the full laser energy. In general, the split ratio will be variable, but for standard operation a 50:50 split ratio is envisioned and each branch is therefore optimized for a maximum pulse energy of $10 \mathrm{~mJ}$.

The diagnostics branch provides the femtosecond pulses for the FEL pulse diagnostics and timing tools, i.e. the photon arrival time and length monitor (PALM) (Juranić et al., 2014a,b) and the spectral encoding (Bionta et al., 2011). The PALM will provide simultaneously, and single-shot, an estimate of the X-ray photon pulse length and an arrival-time measurement between the experimental laser and the X-rays. It is based on the concept of the THz streak camera (Frühling et al., 2009) optimized for the hard X-ray regime (Juranić et al., 2014b). The incident X-ray photons ionize xenon clusters in the presence of an intense THz field. The kinetic energy of the generated electrons varies with the X-rays; the $\mathrm{THz}$ pulse time delay and the variation in the spectral width can be correlated to the X-ray chirp and pulse duration. About $98 \%$ of the energy available for the diagnostics branch will be used to drive the $\mathrm{THz}$ source. It is based on optical rectification with a tilted pulse front (TPF) in $\mathrm{LiNbO}_{3}$ (Hebling et al., 2002), 
optimized for high output energy (Stepanov et al., 2008) or alternatively on BNA (Shalaby et al., 2016). We expect from $\mathrm{LiNbO}_{3}$, with a typical conversion efficiency of $0.1 \%$, a $\mathrm{THz}$ energy of about $10 \mu \mathrm{J}$ and a central frequency of about $0.3-0.5 \mathrm{THz}$. The maximum acceptable time delay between $\mathrm{X}$-rays and $\mathrm{THz}$ is $\pm 350 \mathrm{fs}$. This is limited due to the $\sim 700$ fs-long linear part of the $\mathrm{THz}$ field. The field strength at the interaction point will be $\sim 100 \mathrm{kV} \mathrm{cm}^{-1}$ and the estimated single-shot arrival-time resolution $<10$ fs (Juranić et al., 2014a).

Located after the PALM is an X-ray arrival-time monitor based on spectral encoding of the optical beam. Compared with the $\mathrm{THz}$ streak camera this device is significantly less demanding on input energy and will cover a larger time window (up to $2 \mathrm{ps}$ ). It is based on an X-ray induced refractive index change on a thin (sub-mm) thick $\mathrm{Si}_{3} \mathrm{~N}_{4}$ crystal. From the laser side, a few $\mu \mathrm{J}$ are used to drive the white light generation in a thin sapphire plate and the detection of the output signal on a spectrometer. The chirp is induced by transmission through bulk glass and can be varied by modifying its thickness. With this technique a typical temporal resolution of better than $10 \mathrm{fs}$ has been demonstrated (Harmand et al., 2013).

The experimental branch will provide a predefined set of output options for the users. This ensures limited effort to adjust the optical setup for each beam time and renders fast switching between experiments. The available outputs cover the full spectrum, i.e. UV, visible, NIR and mid-IR, as well as the $\mathrm{THz}$ range. On the same beam path is also attached the LAM for drift stabilizations, which is described in $\$ 5$.

Three basic modes are available (Fig. $2 b$ ). Mode 1 is the direct compressed amplifier output with $<30$ fs pulse duration, $>40 \mathrm{~nm}$ bandwidth, and up to $10 \mathrm{~mJ}$ pulse energy. For mode $2 \mathrm{a}$ the beam is routed through an optical parametric amplifier (8 mJ HE-TOPAS Prime from LightConversion). This allows the up conversion of the $800 \mathrm{~nm}$ pulse to the NIR between $1.2 \mu \mathrm{m}$ and $2.6 \mu \mathrm{m}$ and provides a total pulse energy for signal and idler of up to $2 \mathrm{~mJ}$. To extend the tuning range, several additional wavelength converters will be available (Mode 2b). By routing the beam through the NirUVis module and the NDFG module a spectral range between $240 \mathrm{~nm}$ and $15 \mu \mathrm{m}$ can be accessed. The expected performance of the OPA with the additional converters is summarized in Table 1.

The wavelength range between $15 \mu \mathrm{m}$ and $20 \mu \mathrm{m}$ is not directly accessible with the SwissFEL experimental laser. This requires a 100 fs pump beam, which could be implemented by an adapted stretcher and compressor set on the Ti:sapphire amplifier system. The corresponding space is already allocated.
Mode $2 \mathrm{~b}$ also includes $\mathrm{THz}$ generation by optical rectification in organic crystals (Hauri et al., 2011; Ruchert et al., 2012, 2013). The highly efficient organic nonlinear frequency converters provide radiation between 0.1 and $10 \mathrm{THz}$ and offer a field strength exceeding $1 \mathrm{MV} \mathrm{cm}^{-1}$ (Table 2). The central frequency of the $\mathrm{THz}$ generation can be selected by using different nonlinear materials (Vicario et al., 2015). The $\mathrm{THz}$ generator just needs to be introduced into the collimated OPA output. Compared with $\mathrm{THz}$ generation by TPF in $\mathrm{LiNbO}_{3}$, no sophisticated setup is required. It can be set up next to the experiment to reduce losses due to beam transport. The actual achievable peak field strength might be limited due to the focusing geometry.

Mode 3 will provide ultrashort pulses. The generation is based on nonlinear spectral broadening of an intense Ti:sapphire laser pulse in a gas-filled hollow core fibre (Nisoli et al., 1996, 1997). It will provide up to $500 \mu \mathrm{J}$ pulse energy with sub-10 fs pulse duration, centred around $800 \mathrm{~nm}$.

Due to the initial requirements and scientific layout of the two endstations, the NirUVis module will only be present in ESA. The long-wavelength extensions for differencefrequency generation (DFG) and $\mathrm{THz}$ generation, as well as the few cycle pulse source, will only be available in ESB. Nevertheless, free space will be available in both endstations for users to install their own laser equipment for the experiment.

\section{Laser diagnostics}

The SwissFEL experimental laser consists of many subsystems which all need to operate up to specifications for an experiment to be successful. Thus incidents must be detected and located quickly such that any intervention can directly focus 


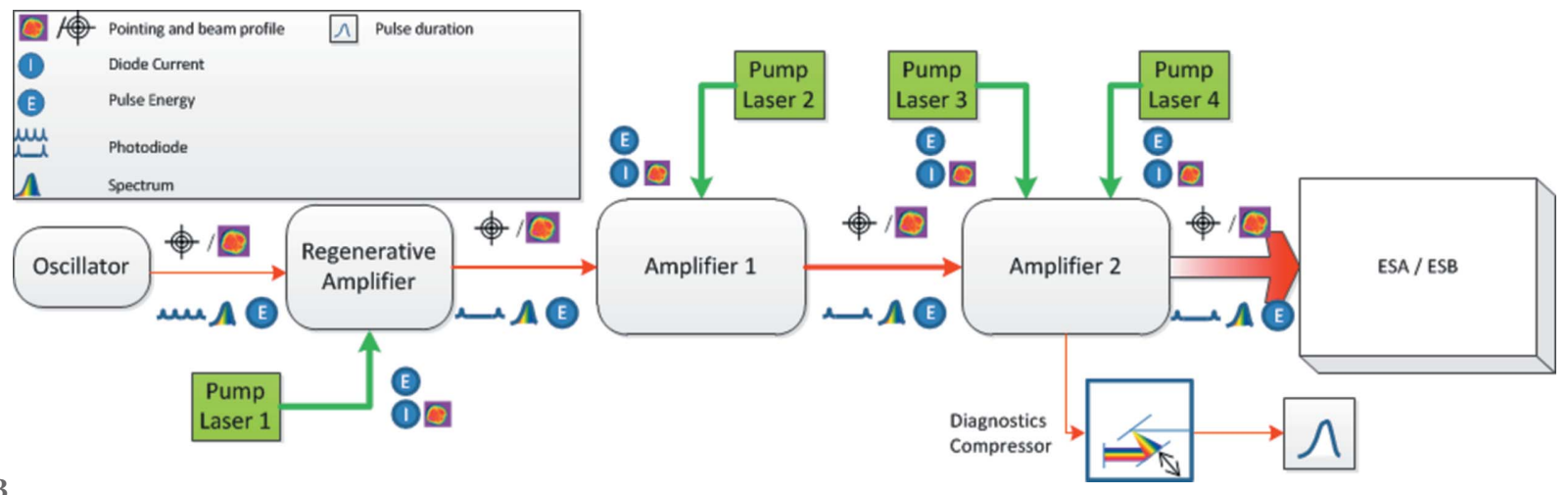

Figure 3

Diagnostics network of the laser amplifier. Oscillator, pump lasers and all amplification stages are equipped with online diagnostics of the essential laser parameters for laser health management and maintenance scheduling.

on the right component and allow the issue to be resolved in a short time period. Therefore a full network of laser diagnostics will be installed along the amplifier chain as well as on the installation in the experimental stations (Figs. 3 and 4), including beam profiling, pulse energy, spectrum, pointing and pulse duration.

All parameters will be recorded on a regular basis and archived in the general archiver database of SwissFEL. This allows monitoring of the long time performance of the individual components as well as planning of scheduled maintenance. For this, the system relies on the installed measurement network (Figs. 3 and 4), as well as information provided by the different components such as laser power supplies, chillers, etc. Where possible, the diagnostics components will be installed to allow for online monitoring by using the leakage of a mirror, or by specific beam splitters. As long as the diagnostic concerns wavelengths of $800 \mathrm{~nm}$ or shorter, the detectors will have single-shot capability, but, as the general aim is to detect drifts, this is not crucial.

A specific set of diagnostics is dedicated to the users and installed close to the experiment (Fig. 4). The main aim is to provide the FEL users with an online measurement of crucial

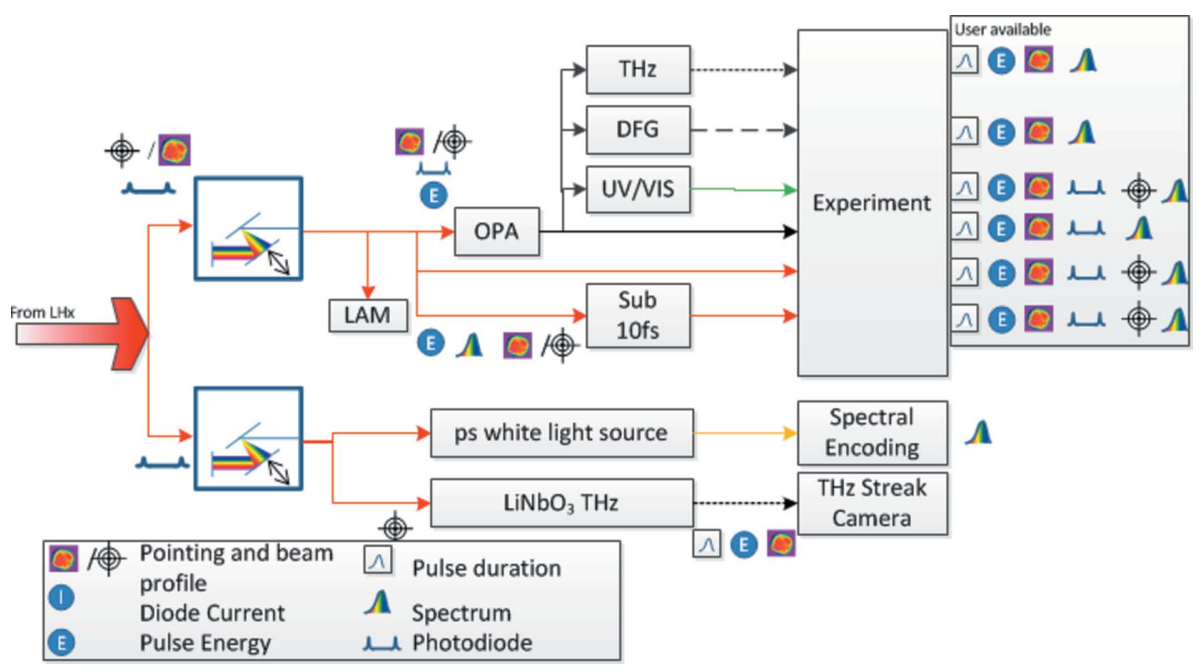

Figure 4

Diagnostics network at the endstation and user-available laser parameters. laser parameters, e.g. pulse energy, pulse duration, pointing, spectrum, etc. For this, the data are fed into the same data stream as the experimental data from the FEL. Ideally this is all single-shot and beam-synchronous with each FEL shot for later correlation analysis. In general, this is possible for $\mathrm{Si}$ based detectors and laser wavelengths below $1100 \mathrm{~nm}$.

For the longer wavelength range above $1 \mu \mathrm{m}$, single-shot acquisition is challenging. With regard to photodiodes, they are still available over a large wavelength range, up to $2 \mu \mathrm{m}$. To cover wavelengths $>2 \mu \mathrm{m}$, mercury cadmium telluride detectors, Golay-cell or microbolometer detectors are typically used. They are relatively slow and the high background noise renders them unsuitable for single-shot acquisition. The only exception is the Shottky diode for frequencies $<2 \mathrm{THz}$ (Semenov et al., 2010), with a rise time of 20 ps. This allows the $\mathrm{THz}$ pulse single-shot to be monitored.

Many single-shot technologies rely on area detectors (e.g. spectrometer, pulse length measurement, e.g. FROG, etc.). Imaging detectors are, to our best knowledge, only available in the wavelength range between $1 \mu \mathrm{m}$ and $2 \mu \mathrm{m}$, based on InGaAs sensors. The resolution and repetition rate is significantly lower than with CMOS- or CCD-based sensors and the cameras are expensive. For the longer wavelength range, pyroelectric detectors are the only option. They are relatively slow and, due to the low-noise performance, not suitable for singleshot acquisition. Thus, additional development effort is required to allow single-shot diagnostics for these cases.

\section{Timing issues}

The synchronization of the laser system to the FEL timing is essential for all pump-probe experiments. In the final configuration the oscillator will be synchronized to the pulsed FEL reference timing system optically, through an optical cross-correlator between the experimental laser and the pulses from the reference timing system, similar to 
the system presented by Schulz et al. (2015). It is expected that the arrival-time jitter between X-rays and experimental laser is $100-150 \mathrm{fs}$ r.m.s. for the beginning of the operation. Since the main limitations are the electron beam parameters and amplitude stability of the accelerating RF structures, it is expected that this value can be reduced towards 20 fs (Ganter, 2010).

The reference timing system is based on pulsed optical links (OneFive, $1550 \mathrm{~nm}$ ) and active length stabilization for drift compensation (Kim et al., 2007, 2008). It is expected that the added timing jitter of this link is $<10 \mathrm{fs}$ r.m.s., with a drift performance of $<10$ fs peak-to-peak over $24 \mathrm{~h}$. Even though these are excellent values, this only locks the oscillator to the reference timing system. The expected timing jitter between oscillator and reference is $<10$ fs r.m.s. $(10 \mathrm{~Hz}$ to $10 \mathrm{MHz})$ (Csatari Divall et al., 2014). After the pulse has left the oscillator it is still propagating through an entire amplifier chain, including a regenerative amplifier, and is sent to the experimental hutches underneath the laser room. The typical optical path length sums up to 50-100 m. Experiment and laser rooms have independent air-conditioning systems and humidity control, which makes the laser pulse highly sensitive to drifts. Already small fluctuations in ambient pressure, humidity and temperature (Ciddor, 1996) give rise to significant laser arrival-time drifts of the order of picoseconds (Csatari Divall et al., 2015). To compensate for these, each endstation is also equipped with a stabilized optical timing link and a LAM is installed on the experimental branch, directly after the compressor. Through spectrally resolved crosscorrelation between the reference timing signal and a weak copy of the amplified pulse, drifts can be measured and compensated. The optical setup of the LAM has a demonstrated resolution of better than $0.3 \mathrm{fs}$. With this technique, the output from a comparable Ti:sapphire amplifier system could be stabilized to $2.36 \mathrm{fs}$ r.m.s. over $10 \mathrm{~h}$, in reference to the direct Ti:sapphire oscillator output. The reconstructed drift between amplifier output and oscillator was exceeding $700 \mathrm{fs}$ over the $10 \mathrm{~h}$ measurement run. The LAM compensates for drifts occurring between the oscillator output and the LAM location in the experimental hutch. The remaining optical path between the LAM and the experiment as well as the FEL timing diagnostics is not covered by the LAM. This leaves a significant part of the beam transport inside the experimental hutch uncontrolled (Fig. 5).

The PALM device measures the time delay between X-rays and the experimental laser at the entrance of the experimental hutches (Fig. 5), while the experiment is taking place $8 \mathrm{~m}$ downstream. The relevant time for the data binning is the arrival time between X-rays and optical laser at the experiment location. To obtain the best accuracy of the arrival-time measurements, the beam path for the experiment (i) needs to be synchronized with the beam path of the diagnostics setup and X-rays [(ii) + (iii)]. The main part of the beam propagation is in free space [(i) and (ii)] and is thus exposed to the environmental changes in the experimental hutch. The X-rays, on the other hand, propagate in a vacuum and are not exposed to the environmental changes. This leaves an optical path

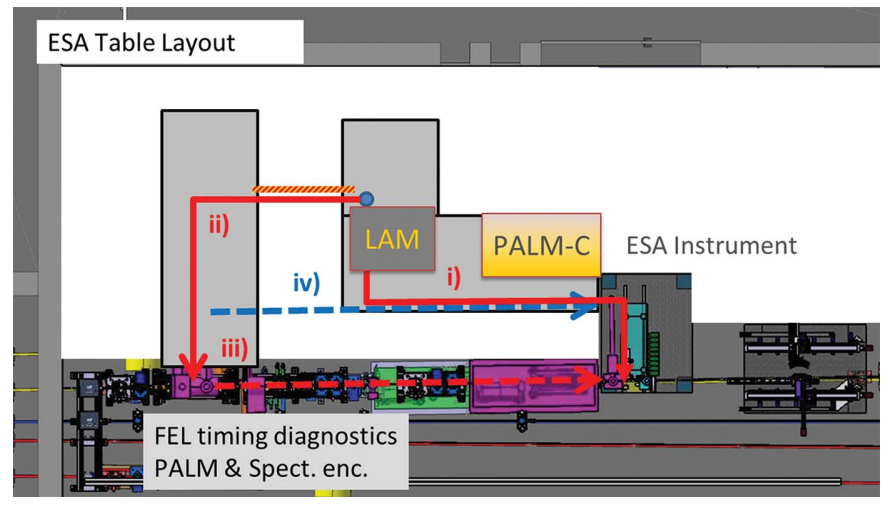

Figure 5

Schematic layout of the beam path on the laser table in ESA with LAM and balanced drift compensator (PALM-C) with (i) the optical beam path of the pulse between the end of the transfer line and the experiment, (ii) the optical beam path from the end of the transfer line to the timing diagnostics, (iii) in-vacuum X-ray beam path between timing diagnostics and experiment, (iv) in-vacuum reference beam path for PALM-C. The same is valid for ESB.

length difference of about $8 \mathrm{~m}$ between the two branches. The pressure dependency of the refractive index in air leads to a group velocity change and induces an arrival time drift of the order of $7 \mathrm{fs} \mathrm{mbar}^{-1}$ (Ciddor, 1996; Edlén, 1966). Thus the delay between the two sources exceeds $70 \mathrm{fs}$ for a minor ambient pressure change of $10 \mathrm{mbar}$. Typical fluctuations during a beam time at SwissFEL (12 h) can reach several tens of mbar. Thus the impact of ambient pressure change becomes significant when operating with ultrashort pulses $(<30 \mathrm{fs})$. Ideally one would therefore measure the laser-X-ray delay directly at the sample position. With the FEL beamline setup this will not be possible and regular recalibration of the arrival time between experiment and PALM is required. To measure these drifts a PALM-Corrector (PALM-C) setup will be installed. PALM-C is based on a balanced optical crosscorrelation between the diagnostics branch and the experimental branch, and is located close to the actual experiment chamber. For this a probe beam is split off from the TPF THz setup and sent through an evacuated tube (iv) towards the end of the experimental branch. The tube has similar length as the $\mathrm{X}$-ray beamline. By this we are mimicking the X-ray propagation along (iii). Since PALM-C has a similar optical layout as the LAM, we expect similar jitter performance and can be directly adapted to the UV-to-NIR range by adapting the phase matching conditions.

\section{Conclusion}

The laser system and infrastructure presented here will build the framework to fulfil the key parameters for user laser operation at SwissFEL, i.e. performance, availability and stability. This is achieved by the combination of choice of laser system, advanced diagnostics and infrastructure. With the presented performance map, a large number of experiments will be possible. When more exotic schemes are required, the provided platform will allow their integration. The key 
advantage, compared with other facilities, will be the achievable timing stability and therefore timing resolution in laserpump/X-ray-probe experiments. The combination of LAM, PALM and PALM-C will provide unprecedented timing resolution on a FEL.

\section{References}

Barty, A., Caleman, C., Aquila, A., Timneanu, N., Lomb, L., White, T. A., Andreasson, J., Arnlund, D., Bajt, S., Barends, T. R. M., Barthelmess, M., Bogan, M. J., Bostedt, C., Bozek, J. D., Coffee, R., Coppola, N., Davidsson, J., DePonte, D. P., Doak, R. B., Ekeberg, T., Elser, V., Epp, S. W., Erk, B., Fleckenstein, H., Foucar, L., Fromme, P., Graafsma, H., Gumprecht, L., Hajdu, J., Hampton, C. Y., Hartmann, R., Hartmann, A., Hauser, G., Hirsemann, H., Holl, P., Hunter, M. S., Johansson, L., Kassemeyer, S., Kimmel, N., Kirian, R. A., Liang, M., Maia, F. R. N. C., Malmerberg, E., Marchesini, S., Martin, A. V., Nass, K., Neutze, R., Reich, C., Rolles, D., Rudek, B., Rudenko, A., Scott, H., Schlichting, I., Schulz, J., Seibert, M. M., Shoeman, R. L., Sierra, R. G., Soltau, H., Spence, J. C. H., Stellato, F., Stern, S., Strüder, L., Ullrich, J., Wang, X., Weidenspointner, G., Weierstall, U., Wunderer, C. B. \& Chapman, H. N. (2012). Nat. Photon. 6, 35-40.

Bertoni, R., Cammarata, M., Lorenc, M., Matar, S. F., Létard, J.-F., Lemke, H. T. \& Collet, E. (2015). Acc. Chem. Res. 48, 774-781.

Bionta, M. R., Lemke, H. T., Cryan, J. P., Glownia, J. M., Bostedt, C., Cammarata, M., Castagna, J.-C., Ding, Y., Fritz, D. M., Fry, A. R., Krzywinski, J., Messerschmidt, M., Schorb, S., Swiggers, M. L. \& Coffee, R. N. (2011). Opt. Express, 19, 21855-21865.

Budarz, J. M., Minitti, M. P., Cofer-Shabica, D. V., Stankus, B., Kirrander, A., Hastings, J. B. \& Weber, P. M. (2016). J. Phys. B, 49, 034001.

Ciddor, P. E. (1996). Appl. Opt. 35, 1566-1573.

Csatari Divall, M., Kaiser, M., Hunziker, S., Vicario, C., Beutner, B., Schietinger, T., Lüthi, M., Pedrozzi, M. \& Hauri, C. P. (2014). Nucl. Instrum. Methods Phys. Res. A, 735, 471-479.

Csatari Divall, M., Mutter, P., Divall, E. J. \& Hauri, C. P. (2015). Opt. Express, 23, 29929-29939.

Danailov, M. B., Cinquegrana, P., Demidovich, A., Ivanov, R., Nikolov, I. \& Siaglotti, P. (2011). Proceedings of the 33rd International Free Electron Laser Conference (FEL2011), 22-26 August 2011, Shanghai, China.

Edlén, B. (1966). Metrologia, 2, 71.

Frühling, U., Wieland, M., Gensch, M., Gebert, T., Schütte, B., Krikunova, M., Kalms, R., Budzyn, F., Grimm, O., Rossbach, J., Plönjes, E. \& Drescher, M. (2009). Nat. Photon. 3, 523-528.

Ganter, R. (2010). SwissFEL Conceptual Design Report. Report 1004. Paul Scherrer Institute, Switzerland.

Glover, T. E., Fritz, D. M., Cammarata, M., Allison, T. K., Coh, S., Feldkamp, J. M., Lemke, H., Zhu, D., Feng, Y., Coffee, R. N., Fuchs, M., Ghimire, S., Chen, J., Shwartz, S., Reis, D. A., Harris, S. E. \& Hastings, J. B. (2012). Nature (London), 488, 603-608.

Harmand, M., Coffee, R., Bionta, M. R., Chollet, M., French, D., Zhu, D., Fritz, D. M., Lemke, H. T., Medvedev, N., Ziaja, B., Toleikis, S. \& Cammarata, M. (2013). Nat. Photon. 7, 215-218.

Hauri, C. P., Ruchert, C., Vicario, C. \& Ardana, F. (2011). Appl. Phys. Lett. 99, 161116.

Hebling, J., Almasi, G., Kozma, I. \& Kuhl, J. (2002). Opt. Express, 10, 1161-1166.

Ingold, G. \& Beaud, P. (2013). SwissFEL ARAMIS Endstation ES-B Conceptual Design Report. Paul Scherrer Institute, Switzerland.

Juranić, P. N., Stepanov, A., Ischebeck, R., Schlott, V., Pradervand, C., Patthey, L., Radović, M., Gorgisyan, I., Rivkin, L., Hauri, C. P., Monoszlai, B., Ivanov, R., Peier, P., Liu, J., Togashi, T., Owada, S., Ogawa, K., Katayama, T., Yabashi, M. \& Abela, R. (2014a). Opt. Express, 22, 30004-30012.
Juranić, P. N., Stepanov, A., Peier, P., Hauri, C. P., Ischebeck, R., Schlott, V., Radović, M., Erny, C., Ardana-Lamas, F., Monoszlai, B., Gorgisyan, I., Patthey, L. \& Abela, R. (2014b). J. Instrum. 9, P03006.

Kim, J., Chen, J., Zhang, Z., Wong, F. N. C., Kärtner, F. X., Loehl, F. \& Schlarb, H. (2007). Opt. Lett. 32, 1044-1046.

Kim, J., Cox, J. A., Chen, J. \& Kärtner, F. X. (2008). Nat. Photon. 2, 733-736.

Kim, K. H., Kim, J. G., Nozawa, S., Sato, T., Oang, K. Y., Kim, T. W., Ki, H., Jo, J., Park, S., Song, C., Sato, T., Ogawa, K., Togashi, T., Tono, K., Yabashi, M., Ishikawa, T., Kim, J., Ryoo, R., Kim, J., Ihee, H. \& Adachi, S. (2015). Nature (London), 518, 385389.

Kubacka, T., Johnson, J. A., Hoffmann, M. C., Vicario, C., de Jong, S., Beaud, P., Grübel, S., Huang, S.-W., -, W., Huber, L., Patthey, L., Chuang, Y., -, D., Turner, J. J., Dakovski, G. L., Lee, W., -, S., Minitti, M. P., Schlotter, W., Moore, R. G., Hauri, C. P., Koohpayeh, S. M., Scagnoli, V., Ingold, G., Johnson, S. L. \& Staub, U. (2014). Science, 343, 1333-1336.

Lemke, H. T., Bressler, C., Chen, L. X., Fritz, D. M., Gaffney, K. J., Galler, A., Gawelda, W., Haldrup, K., Hartsock, R. W., Ihee, H., Kim, J., Kim, K. H., Lee, J. H., Nielsen, M. M., Stickrath, A. B., Zhang, W., Zhu, D. \& Cammarata, M. (2013). J. Phys. Chem. A, 117, 735-740.

Lévy, A., Audebert, P., Shepherd, R., Dunn, J., Cammarata, M., Ciricosta, O., Deneuville, F., Dorchies, F., Fajardo, M., Fourment, C., Fritz, D., Fuchs, J., Gaudin, J., Gauthier, M., Graf, A., Lee, H. J., Lemke, H., Nagler, B., Park, J., Peyrusse, O., Steel, A. B., Vinko, S. M., Wark, J. S., Williams, G. O., Zhu, D. \& Lee, R. W. (2015). Phys. Plasmas, 22, 030703.

Mankowsky, R., Subedi, A., Först, M., Mariager, S. O., Chollet, M., Lemke, H. T., Robinson, J. S., Glownia, J. M., Minitti, M. P., Frano, A., Fechner, M., Spaldin, N. A., Loew, T., Keimer, B., Georges, A. \& Cavalleri, A. (2014). Nature (London), 516, 71-73.

Milne, C. J. (2013). SwissFEL Experimental Station A: Conceptual Design Report. Paul Scherrer Institute, Switzerland.

Minitti, M. P., Robinson, J. S., Coffee, R. N., Edstrom, S., Gilevich, S., Glownia, J. M., Granados, E., Hering, P., Hoffmann, M. C., Miahnahri, A., Milathianaki, D., Polzin, W., Ratner, D., Tavella, F., Vetter, S., Welch, M., White, W. E. \& Fry, A. R. (2015). J. Synchrotron Rad. 22, 526-531.

Nisoli, M., De Silvestri, S. \& Svelto, O. (1996). Appl. Phys. Lett. 68, 2793-2795.

Nisoli, M., Stagira, S., De Silvestri, S., Svelto, O., Sartania, S., Cheng, Z., Lenzner, M., Spielmann, C. \& Krausz, F. (1997). Appl. Phys. B, 65, 189-196.

Prat, E., Löhl, F. \& Reiche, S. (2015). Phys. Rev. ST Accel. Beams, 18, 100701.

Prat, E. \& Reiche, S. (2015). Phys. Rev. Lett. 114, 244801.

Rettig, L., Mariager, S. O., Ferrer, A., Grübel, S., Johnson, J. A., Rittmann, J., Wolf, T., Johnson, S. L., Ingold, G., Beaud, P. \& Staub, U. (2015). Phys. Rev. Lett. 114, 067402.

Ruchert, C., Vicario, C. \& Hauri, C. P. (2012). Opt. Lett. 37, 899901.

Ruchert, C., Vicario, C. \& Hauri, C. P. (2013). Phys. Rev. Lett. 110, 123902.

Schulz, S., Grguraš, I., Behrens, C., Bromberger, H., Costello, J. T., Czwalinna, M. K., Felber, M., Hoffmann, M. C., Ilchen, M., Liu, H. Y., Mazza, T., Meyer, M., Pfeiffer, S., Prędki, P., Schefer, S., Schmidt, C., Wegner, U., Schlarb, H. \& Cavalieri, A. L. (2015). Nat. Commun. 6, 5938.

Semenov, A., Cojocari, O., Hübers, H.-W., Song, F., Klushin, A. \& Müller, A.-S. (2010). IEEE Electron Device Lett. 31, 674-676.

Shalaby, M. \& Hauri, C. P. (2015). Nat. Commun. 6, 5976.

Shalaby, M., Vicario, C., Thirupugalmani, K., Brahadeeswaran, S. \& Hauri, C. P. (2016). Opt. Lett. 41, 1777-1780.

Shwartz, S., Fuchs, M., Hastings, J. B., Inubushi, Y., Ishikawa, T., Katayama, T., Reis, D. A., Sato, T., Tono, K., Yabashi, M., 
Yudovich, S. \& Harris, S. E. (2014). Phys. Rev. Lett. 112, 163901.

Stanislauskas, T., Budriūnas, R., Antipenkov, R., Zaukevičius, A., Adamonis, J., Michailovas, A., Giniūnas, L., Danielius, R., Piskarskas, A. \& Varanavičius, A. (2014). Opt. Express, 22, 1865-1870.

Stepanov, A. G., Bonacina, L., Chekalin, S. V. \& Wolf, J.-P. (2008). Opt. Lett. 33, 2497-2499.

Tavella, F., Nomura, Y., Veisz, L., Pervak, V., Marcinkevičius, A. \& Krausz, F. (2007). Opt. Lett. 32, 2227.

Telle, H. R., Steinmeyer, G., Dunlop, A. E., Stenger, J., Sutter, D. H. \& Keller, U. (1999). Appl. Phys. B, 69, 327-332.

Tono, K., Togashi, T., Inubushi, Y., Sato, T., Katayama, T., Ogawa, K., Ohashi, H., Kimura, H., Takahashi, S., Takeshita, K., Tomizawa, H.,
Goto, S., Ishikawa, T. \& Yabashi, M. (2013). New J. Phys. 15, 083035 .

Trisorio, A., Paul, P. M., Ple, F., Ruchert, C., Vicario, C. \& Hauri, C. P. (2011). Opt. Express, 19, 20128-20140.

Vicario, C., Ruchert, C. \& Hauri, C. P. (2015). J. Mod. Opt. 62, 14801485.

Young, L., Kanter, E. P., Krässig, B., Li, Y., March, A. M., Pratt, S. T., Santra, R., Southworth, S. H., Rohringer, N., Dimauro, L. F., Doumy, G., Roedig, C. A., Berrah, N., Fang, L., Hoener, M., Bucksbaum, P. H., Cryan, J. P., Ghimire, S., Glownia, J. M., Reis, D. A., Bozek, J. D., Bostedt, C. \& Messerschmidt, M. (2010). Nature (London), 466, 56-61.

Zewail, A. H. (2000). J. Phys. Chem. A, 104, 5660-5694. 